\title{
Editorial
}

\section{NOVEL CORONAVIRUS VULNERABILITY: METABOLIC DERANGEMENTS AND ALTERED DEHYDROGENASE ENZYME ACTIVITIES ARE CENTRAL TO THE VIRUS PATHOGENESIS IN HUMAN, BAT AND OTHER ANIMALS}

\author{
Sanjib Kumar Manna*
}

\begin{abstract}
The ongoing pandemic of novel coronavirus disease (COVID-19) has killed millions across the globe. This paper hypothesizes anaerobic glycolysis and dehydrogenase enzymes as the focal points of SARS-CoV-2 pathogenesis that ensure virus survival and replication in humans and the reservoir host bat. Metabolic alterations in hypertension, diabetes, obesity, and higher reliance on anaerobic glycolysis for energy generation make males and aged people more vulnerable to the disease. The bat has a low level of vitamin D, greater metabolic dependence on anaerobic glycolysis, and low dehydrogenase activities which might predispose this mammal to persistent infection. Similarities of the bat metabolism with the metabolic changes brought by SARS-CoV-2 in humans suggest possible evolution of the coronavirus targeting the host metabolic processes for its replication and survival. Further research on host-pathogen interactions at the metabolism levels would unravel the pathogenesis of coronavirus and several other viruses.
\end{abstract}

Key words: Covid -19, Vulnerability, Species, Age, Sex, Comorbidity.

The novel coronavirus disease (COVID-19), caused by highly infectious SARS-CoV-2, has caused a global public health emergency sickening more than 177 million people so far (https://coronavirus.jhu.edu). The disease affects the lungs and several other organs giving rise to diverse symptoms and complications extending for months or even more. Men and elderly people are significantly more prone to the infection and death (Grasselli et al. 2020). Underlying conditions such as type 2 diabetes, obesity, hypertension, cardiovascular diseases, chronic respiratory problems, kidney damage, and even ethnicity influence the disease outcome (Grasselli et al. 2020, Smith et al. 2020).

The disease progression and fatality of SARS-CoV-2 have been explained in terms of distribution and functions of the cell receptor angiotensin-converting enzyme 2 (ACE2) and the renin-angiotensin system (RAS). ACE2 is highly expressed in the lungs, blood vessels, and also in intestine and kidneys frequently linking signs and outcomes to these organs (Gheblawi et al. 2020). ACE2 cleaves vasoconstrictive angiotensin-II (Ang II) to Ang(1-7) and thus is a negative regulator of RAS and critical for blood vascular homeostasis (Hardtner et al. 2013). Depletion of antithrombotic ACE2 at the cell surface with the binding of the coronavirus causes over-activation of
RAS and inflammatory cytokines resulting in unregulated intravascular coagulation in the lungs and other tissues in severe cases (Gheblawi et al. 2020). However, ACE2 alone does not explain the age and sex biases of COVID19 since the receptor expression is neither different among men and women, nor it increases with age (Xie et al. 2006, Li et al. 2020). This paper hypothesizes altered dehydrogenase enzymes and upregulated anaerobic glycolysis as the most important pathogenic mechanisms of SARS-CoV-2 which influence the undesired outcome in comorbid patients, old age, etc., and also unite the virus with its reservoir host.

\section{ACE2 is involved in energy generation pathways}

ACE2, an important component of alternative RAS axes, is part of the body's glucose homeostasis mechanism executed through insulin production, secretion, and insulin-mediated glucose uptake (Batlle et al. 2010, Hardtner et al. 2013). RAS activation with loss of ACE2 is linked to altered glucose metabolism (Gheblawi et al. 2020). The expression of ACE2 is epigenetically regulated and influenced by cellular energy deprivation and stress such as hypoxia, increased AMP, and IL-1 $\beta$ levels (Clarke et al. 2014). Thus, the expressions of ACE2,

*Principal Scientist, ICAR-Central Inland Fisheries Research Institute, Barrackpore, India - 700120. 
inflammatory cytokines, and RAS activation are influenced by the glucose-linked energy homeostasis of the body. ACE2 has a strong protective role in pulmonary epithelium: higher ACE2 levels are noted in the lungs of individuals with pulmonary diseases, presumably due to higher levels of oxidative stress which make them more vulnerable to COVID-19 (Pinto et al. 2020). Dysregulated expression of proinflammatory cytokine interleukin-6 (IL6) by hyperactive immune cells has also been linked to low ACE2 and energy levels (Gheblawi et al. 2020). All these suggest a close link between body energetic and SARS-CoV-2 pathogenesis.

\section{SARS-COV-2 alters cell metabolism, especially glycolysis}

An increasing number of researchers are emphasizing metabolic remodelling of host cells by SARS-CoV-2. Clinical profiles of COVID-19 patients show hyperglycaemia, insulin resistance as well as dyslipidaemia (Wu et al. 2020, Ehrlich et al. 2020). The virus also induces alterations in mitochondrial structure and functions, including the Kreb's cycle and oxidative phosphorylation (Ehrlich et al. 2020, Singh et al. 2020). A plasma metabolomic and lipidomic study shows disturbed whole-body carbon, urea, and pyrimidine metabolisms (Wu et al. 2020). Among several metabolic alterations by the virus, glucose metabolism, particularly glycolysis, is grossly deranged; amino acid metabolism, with a lesser role in cellular energetic, is not altered significantly (Ehrlich et al. 2020). Moderate and severe COVID-19 is associated with dysregulated glucose metabolism expressed as new and persistent hyperglycaemia (Smith et al. 2020). The infected lung epithelium shows strong metabolic transcriptional responses, notably suppressed Kreb's cycle and oxidative phosphorylation, and upregulation of pyruvate to lactate synthesis (anaerobic glycolysis, AnG), pentose phosphate pathway (PPP), and lipogenesis towards nucleotide and cholesterol synthesis favouring virus replication. Infected cells rely more on lactate over glucose oxidation and overexpress genes involved in AnG (Ehrlich et al. 2020). Some of the other viruses such as adenovirus, human cytomegalovirus, herpes simplex virus-1, Kaposi's sarcoma-associated herpesvirus also cause diversion of glycolysis (Mayer et al. 2019), however, there might be virus-specific pattern of metabolic hijacking in qualitative and quantitative terms which needs further study.

Decoupling of mitochondrial respiration results in oxidative stress commonly seen in severe cases in COVID-19 (Ehrlich et al. 2020, Codo et al. 2020). Metabolic diversion from oxidative phosphorylation to
AnG and PPP results in less ATP generation per unit of glucose and is a likely cause of extreme weakness in COVID-19 patients. Reduced energy generation is also evident from a significant fall in mitochondrial membrane potential $((\Delta \psi \mathrm{m})$ in infected cells (Ehrlich et al. 2020). Mitochondrial inhibition and diversion of pyruvate to lactate results in reduced glucose oxidation to $\mathrm{CO}_{2}$ with resultant glucose build-up and severe hyperglycaemia. Codo et al. (2020) observed that glycolysis flux and higher glucose concentrations directly enhanced the virus load in infected monocytes and expressions of ACE2, and pro-inflammatory cytokines IL- $1 \beta$, IL-6, TNF- $\propto$ causing the cytokine burst. Reduced levels of interferon (IFN) and elevated levels of chemokines and IL-6 lead to the development of severe COVID-19 (Codo et al. 2020, Blanco-Melo et al. 2020). Research also shows close links between diabetes, obesity, and IL-6 expression (Timper et al. 2017); hyperglycaemia acts as a trigger for over-activation of inflammatory cytokines and abnormal intravascular coagulation (Randeria et al. 2019). It is thus not surprising that these metabolic diseases have been associated with the severity of COVID-19.

\section{Dehydrogenase enzymes - another target of corona virus}

Dehydrogenase (DHase) enzymes are critically important in energy generation, besides several other biochemical functions. The virus upregulates the expression of lactate dehydrogenase (LDH) enzyme (Ehrlich et al. 2020). Clinically, serum LDH enzyme level increases many-folds and is a predictor of severe COVID19 (Henry et al. 2020). LDH converts pyruvic acid to lactate and NADH to $\mathrm{NAD}^{+}$- this NADH recycling enables the glycolysis pathway to continue at a faster pace, and, in this case, synthesizing building blocks for virus. Besides LDH, alterations of several other DHase enzymes of glucose and lipid metabolism have been observed in SARS-CoV-2 infection (Ehrlich et al. 2020). Glyceraldehyde-3-phosphate dehydrogenase (GAPDH) mRNA levels were highly correlated with SARS-CoV positivity (Wong et al. 2005). Inosine monophosphate dehydrogenase inhibitors such as ribavirin enhance the infectivity of SARS-CoV suggesting roles of DHase enzymes in protection against coronavirus (Barnard et al. 2006). Glucose-6-phosphate dehydrogenase (G6PD) is critical for cell survival against coronavirus and several other virus infections and G6PD deficiency enhances human coronavirus (HCoV) 229E infection (Wu et al. 2008). G6PD is involved in the production of NADPH and glutathione metabolism which protect cells against 
redox-mediated damages. G6PD deficiency has been detected among paediatric cases of COVID-19 (Al-Aamri et al. 2020). Genetic G6PD deficiency is again associated with a higher prevalence of diabetes and hypertension, the most important comorbidities of COVID-19 (Gaskin and Reddy 2001). From the demographic distribution of genetic G6PD deficiency among the Mediterranean, African, Asian populations and COVID-19 fatality rates, G6PD deficiency has been predicted as a predisposing factor to severe COVID-19 (Vick 2020, Jamerson et al. 2020).

\section{Metabolic signature of the bat}

The SARS-CoV-2 has been suggested to originate from the bat, the only flying mammal. Fruit bat consumes a large amount of sugar which is rapidly absorbed from the gut and becomes available for oxidation by a strong insulin response from an exceptionally well-developed endocrine pancreas (Protzek et al. 2010, Meng et al. 2016). Despite its ability to maintain glucose homeostasis, significantly high blood glucose and lactate levels indicate strong anaerobic respiration in bat (Meng et al. 2016, Pinheiro et al. 2006). Comparative evaluation of succinate dehydrogenase (SDH) and LDH enzyme activities of the small intestine indicates that the bat prefers AnG over Kreb's cycle to meet the energy demands (Ofusori et al. 2007). Despite higher sugar intake, the bat has the lowest G6PD and higher LDH enzyme activities suggesting low pentose phosphate pathway (PPP) and relatively high AnG activities among the three nocturnal mammals, viz. frugivorous bat, omnivorous rat, and insectivorous pangolin (Adeniyl et al. 2012). Thus, from the above three works together (Protzek et al. 2010, Ofusori et al. 2007, Adeniyl et al. 2012) compromised PPP and mitochondrial oxidation, and compensatory high AnG in bat are evident. High contents of LDH enzymes have been detected in skeletal muscles of bat which, like other mammals, use the lactate pathway for burst of energy generation for flying (Thomas and Suthers 1972, Antonova et al. 2018). Bat has other adaptations also for flying: it has reduced its genome size and lost a number of genes involved in immune function including IFN locus copy numbers (Zhou et al. 2016). An experimental infectivity study has shown accelerated dynamics of replication-competent vesicular stomatitis Indiana viruses in bat cell lines even in presence of IFN- $\propto$, IFN- $\beta$ responses (Brook et al. 2020). Bat has developed a constitutive yet dampened IFN response and other antiviral defence mechanisms (Brook et al. 2020 and references therein) enabling fast virus dynamics and downplayed its inflammatory response for survival leading to long term viral persistence
(Brook et al. 2020, Gorbunova et al. 2020, Pennisi 2020). Mitochondria and its active electron transport chain play central roles in signalling pathways and production of pro-inflammatory cytokines and other innate immunity effectors such as IFNs, NF-KB, IL-6, C11 orf83, RNaseL, IRFs, etc. (Yang et al. 2017, and references therein). SARS-CoV-2 has been found to mute reactive oxygen species (ROS)-mediated mitochondrial antiviral signalling (Miller et al. 2021) that can potentially enhance the chance of virus survival in Chiropteran and nonChiropteran mammals. It is plausible that compromised mitochondrial innate immune response, suboptimal Kreb's cycle and PPP, and higher dependence on AnG provide a safe and congenial environment for rapid virus replication and propagation in bats making it a natural reservoir of coronavirus and several other communicable viruses. Strong similarities of bat's metabolic signatures, viz., high AnG, low mitochondrial respiration, and G6PD activities with similar clinical profiles of COVID-19 patients are noteworthy, which also suggests that the virus might have evolutionarily developed mechanisms to modulate the host metabolic processes to replicate and weaken cell defence for long term persistence.

\section{Metabolic soundness and the type of metabolism are the keys against SARS-COV-2}

Metabolism is the central theme of every living cell for need-based energy generation, and synthesis of different monomeric molecules as building blocks of cells and tissues. To meet the changing energy and growth requirements at different age body metabolism undergoes multiple shifts in one's lifetime (Ravera et al. 2019). Sugar/glucose is the major energy source in humans. During infancy and childhood, glucose-6-phosphate (G6P) is majorly diverted to PPP, instead of pyruvic acid, to synthesize NADPH, fatty acids, nucleic acids, nucleotides, etc. for faster body growth (Hakim et al. 1980, Niedermuller 1986). This high synthesis activity, along with subsistence level of mitochondrial respiration and resultant low innate cell defence (Ravera et al. 2019, Yang et al. 2017) might make infants and kids susceptible to SARS-CoV-2. However, abundant production of reducing NADPH and a low level of AnG protect this age group from developing a severe form of the disease. The growth requirements decrease, and energy demands increase as we mature and cells channelize G6P through pyruvic acid generating 2 ATP per glucose molecule, and then completely oxidize it in Kreb's cycle to generate more ATP through oxidative phosphorylation (van Beek et al. 2016, Ravera et al. 2019). I hypothesize that very high metabolic rate (BMR) along with fully active 
glycolysis and mitochondrial respiration producing the highest level of energy and innate immune effectors make the teenagers and young adults more resistant to the infection and development of the overt disease.

Glycolysis slows down with age to avoid the formation of toxic intermediates and cells progressively shift to mitochondrial respiration using fatty acids, and to lesser extents, amino acids (Dong and Brewer 2019, van Beek et al. 2016, Fussell et al. 2016). Mitochondrial respiration also decreases in the elderly leading to incomplete utilization of $\mathrm{NADH} / \mathrm{H}^{+}$and respiratory oxygen generating a smaller number of ATP but more of ROS the hallmark of oxidative stress and aging (Ravera et al. 2019). PPP fast slows down with age (Hakim et al. 1980, Ravera et al. 2019, Niedermuller 1986) producing less NADPH and leaving cells less protected against oxidative damage. Deficiency of G6PD, a key enzyme in PPP, among the Mediterranean populations (Vick 2020, Jamerson et al. 2020) or low-level activity in bat (Adeniyl et al. 2012) thus enhance susceptibility or vulnerability to SARS-CoV-2 infection. When cells cannot generate enough energy through Kreb's cycle due to aging-related metabolic slow-down or low level of lipolysis they shift back to glycolysis, but this time to AnG even in presence of oxygen (Warburg effect) (Garcia-Alvarez et al. 2014, Liberti and Locasale 2016, Ravera et al. 2019). AnG peaks from the $3^{\text {rd }}$ and $4^{\text {th }}$ decade of life (Ravera et al. 2019). Highly upregulated AnG, weak mitochondrial respiration, and low NADPH production in greying and old ages (van Beek et al. 2016, Ravera et al. 2019) strongly favour SARS-CoV-2 infection, which in presence of a high level of oxidative stress cause severe form of the disease.

Vitamin D deficiency has been associated with COVID-19 infection and fatality, especially among the elderly, and the population having higher skin melanin contents in temperate countries (Lanham-New et al. 2020). Vitamin D supplementation has clinically proven efficacy in recovery from the disease (Rastogi et al. 2020). It influences basal metabolism and several biochemical processes and stabilizes expressions of IL-6 (Bikle 2020, Jain et al. 2020). The sunshine vitamin is also known to reprogram AnG in favour of reductive power and protection against oxidative stress; it restrains AnG through down-regulation of $\mathrm{LDHa}$ (converts pyruvate to lactate) and upregulation of $L D H b$ gene (converts lactate to pyruvate) besides influencing several other genes of the glycolysis and polyol pathways (Santos et al. 2017). The LDH enzyme activity in the small intestinal epithelium of vitamin D deficient rat was significantly less than in normal animals and administration of vitamin D quickly restored the enzyme activity (Nasr et al. 1989).
Vitamin D administration has also been found to restore altered serum LDH in rats with diabetic cardiomyopathy showing the role of the sunshine vitamin in the restoration of DHase enzymes (Zeng et al. 2017). The bat has a very low level of circulating vitamin D (Cavaleros et al. 2003) and this may be correlated with its AnG and low DHase activities. Whether vitamin $\mathrm{D}$ protects against coronavirus partly by strengthening of LDH and other DHase activity remains to be confirmed.

Transmission of SARS-CoV-2 has been documented in minks, domestic and wild cats, dogs, and few other species with low morbidity and fatality rates, except in minks where the disease can be severe (Hosie et al. 2021, CDC 2021). Protein is a major source of energy in strict carnivores which might challenge the above glucosedependent hypothesis of SARS-CoV-2 vulnerability. Nonetheless, glucose metabolism is important in carnivores also. Minks efficiently use carbohydrates with a glucose turnover rate of $4-5 \%$ per minute (Fink and Borsting 2002). Cats have active gluconeogenesis to synthesize glucose for organs like the brain. Interestingly, fasting cats, dolphins develop severe and persistent hyperglycaemia, similar to what happens in diabetes and in COVID-19 patients, through gluconeogenesis and glycogenolysis indicating glucose as a potential energy substrate in energy deficiency states; however, longlasting hyperglycaemia suggests slow rate of glucose oxidation (Schermerhorn 2013). "Stress hyperglycaemia" with increased lactate synthesis is also common in cats (Rand et al. 2002). Interestingly, cat leucocytes have higher hexokinase and LDH enzyme activities than in dogs (Washizu et al. 1998). Serum lactate levels of healthy cats and dogs are considerably high which may increase severely in sick animals suggesting AnG an important metabolic pathway in these animals (Allen et al. 2008, Redavid et al. 2016).

Lactate synthesis by skeletal muscle during heavy exercise is well known. Although glycolysis and AnG generate a smaller number of ATP molecules, the energy generation is about 10-100 times faster than when glucose is completely oxidized in Kreb's cycle, through a massive expression of genes of the glycolysis pathway and fast recycling of $\mathrm{NAD}^{+}$by the $\mathrm{LDH}$ enzyme (Liberti and Locasale 2016). Thus glycolysis, especially AnG, provides a burst of energy to skeletal muscles enabling intense exercise, sprint, fast run for predation, fight and flight responses etc. (Sullivan and Somero 1980, Melendez-Morales et al. 2009). AnG is the major metabolic pathway of bats for fast energy generation during flying (Thomas and Suthers 1972). Anaerobic glycolysis strongly favours SARS-CoV-2 replication 
(Codo et al. 2020) and use of AnG for predation, fight etc. might make the carnivores susceptible to mild to severe form of SARS-CoV-2 infection. Birds frequently use glycolysis during a sprint (Melendez-Morales et al. 2009), making it another naturally susceptible host of coronavirus.

Fatty acids contribute to life expectancy and are important energy sources since around middle age (Fussell et al. 2016, Johnson and Stolzing 2019). Individuals with a low level of fat oxidation would generate less energy, especially in old age when the rate of glucose oxidation is already low. Obese people, having a low-fat oxidation rate, are thus more vulnerable to COVID-19. Lipolysis enhancing drugs such as statin, fenofibrate reverse the altered glycolysis and lipid accumulation and reduce viral load in lung epithelium (Ehrlich et al. 2020). Bioactive lipids, exogenous ketone supplementation have also been suggested to prevent cytokine bursts (Bradshaw et al. 2020, Ghaffari et al. 2020). Excess sugar intake not only provides a ready substrate for glycolysis, it would also not allow sufficient fatty acid oxidation to proceed. Such over-dependence of adults on sugars for energy and inhibition of mitochondrial respiration by SARS-CoV-2 would reduce body energy to critically low levels among the middle aged and elderly and patients with underlying metabolic diseases making them more vulnerable to COVID-19 fatality.

Different organs have different energy preferences. Since SARS-CoV-2 relies on glycolysis for replication, it is natural that organs with high rate of glucose turnover, such as the lungs, brain, digestive system, and kidneys would be more vulnerable to the virus. Lungs have an extremely high rate of glucose utilization that can surpass that of brain and kidney (Liu and Summer 2019). Hyperoxia also exposes the lungs to high oxidative damages making them highly vulnerable to cytokine burst.

Besides the integrity of glycolysis and Kreb's cycle, overall metabolic rate of an individual might play important roles against the SARS-CoV-2 infection. Both the infection and death rates from COVID-19 are low among growing children/young adults and higher in elderly people, which may be correlated with age-related variations in BMR and energy homeostasis (Lazzer et al. 2012, Ravera et al. 2019). The influence of ACE2 gene therapy on glucose metabolism declined in old $d b / d b$ mice due to a general decline in cellular metabolism, including gene expressions and homeostatic responses with age (Batlle et al. 2010). The current worldwide COVID-19 case fatality rate of about $2.1 \%$ (https:// coronavirus.jhu.edu) is much less than nearly $20 \%$ fatality in the Spanish influenza pandemic, $10 \%$ in SARS, and $34 \%$ in MERS indicating that the SARS-CoV-2 is comparatively less lethal. It is assumed that teenagers and young adults with high BMR and strong glycolysisKreb's cycle axis can resist or overcome the infection while the elderly and persons with underlying metabolic diseases succumb to this less virulent virus.

Diabetes mellitus, hypertension, and obesity are associated with reduced or altered body metabolism at multiple levels, and ACE2/Ang II deregulation making these metabolic or lifestyle diseases important comorbidities of COVID-19 (Grasselli et al. 2020). There is impaired glucose uptake and oxidation, and dysregulated glycolysis in diabetes (Guo et al. 2012). Obesity is associated with several metabolic changes including higher anaerobic glycolysis and reduced oxidation of body fat (Uranga and Keller 2019). Hypertensive subjects show whole-body metabolic alterations such as hyperglycaemia, type 2 diabetes, and hyperlipidaemia (Polak-Iwaniuk et al. 2019). These metabolic insufficiencies reduce the body's steady-state energy levels favouring the onset of the cytokine storm.

In absence of long-term data, it is not yet established whether climate or season influences SARS-CoV-2 epidemiology or not, but the virus has defied a general rule of higher winter prevalence of flu and other respiratory virus diseases. Different countries have recorded higher COVID-19 cases in different seasons, but winter peaks in temperate countries and summer peaks in tropical countries, including India, are notable. Environmental stress such as extreme heat or cold enhance glycolysis especially AnG, compromise mitochondrial respiration and fatty acid oxidation (Bouchama et al. 2017, Sun et al. 2019, Chang et al. 2020) which are also the metabolic signature in several other stresses, including oxidative stress. Although oxidative stress is linked to SARS-CoV-2 pathogenesis, further studies are required to establish clear links between climatic stresses with the disease.

\section{Metabolic differences between male and female and predisposition to COVID-19}

Like age, there are sex-related differences in metabolism too. A female has higher pyruvate, and betaoxidation than a male who has a high capacity for AnG (Gaignard et al. 2015, Demarest and McCarthy 2015) these metabolic characteristics might make men more suitable and women less suitable hosts for the virus replication. Mitochondrial functioning, including electron transport chain and ATP levels, are significantly higher 
in females (Silaidos et al. 2018). Males also have higher deleterious mutations in the mitochondrial genome and are more prone to mitochondrial dysfunction (Demarest and McCarthy 2015, Marais et al. 2018) that might predispose them more to an eventful outcome of COVID19.

Active mitochondrial respiration oxidizing glucose and fatty acids are critical for protection against the virus. Boosting body metabolism, energy levels, and antioxidant power with the use of vitamin $\mathrm{D}$, foods rich in readily oxidizable fatty acids, ù- 3 fatty acids and antioxidants, fibre-rich foods for enhancing fatty acid availability through fermentative digestion, therapeutic interventions targeting lipid breakdown or reducing glucose bioavailability, reducing intake of high glycaemic foods including sugar, reducing stress, etc. might help prevent infection, minimize the impact of COVID-19 and perhaps save lives. Fasting limits sugar availability, enhances lipolysis (Torchon et al. 2017) as well as mitochondrial biogenesis (Mehrabani et al. 2020) and thus periodic fasting by healthy adults might protect from a severe form of COVID-19. Recent vaccine availability and vaccination drive by several countries have raised global hope against the disease. Yet, focusing research on virushost interactions at the levels of cell metabolism, energetic and cell defence might provide valuable information towards control of infectious diseases at present and in years to come.

\section{REFERENCES}

Adeniyl TD, Adekomi DA, Tinaji AA, Akinyinka AO (2012) Histochemical evaluation of the activities of glucose-6phosphate dehydrogenase (G-6-PDH) and lactate dehydrogenase (LDH) in the visual relay centers of rat (Rattus norvegicus), bat (Eidolon helvum) and pangolin (Manis tricuspis). J Cell Anim Biol 6(13):188-191.

Al-Aamri M, Al-Khalifa F, Al-Nahwi F, Al-Ameer H, AlAbdi S (2020) G6PD deficiency overrepresented among pediatric COVID-19 cases in one Saudi Children Hospital. medRxiv, https://doi.org/10.1101/2020.07.08.20148700.

Allen SE, Holm JL (2008) Lactate: physiology and clinical utility. J Vet Emerg Crit Care 18(2): 123-132.

Antonova EP, Ilyukha VA, Sergina SN, Unzhakov AR, Belkin VV (2018) Lactate dehydrogenase isoenzymes in the tissues of hibernating bats. Biophys 63: 116-121.

Barnard DL, Day CW, Bailey K, Heiner M, Montgomery R et al. (2006) Enhancement of the infectivity of SARS-CoV in
BALB/c mice by IMP dehydrogenase inhibitors, including ribavirin. Antivir Res 71: 53-63.

Batlle D, Soler MJ, Ye M (2010) ACE2 and diabetes: ACE of ACEs? Diabetes 59:2994-2996.

Bikle DD (2020) Vitamin D: Newer concepts of its metabolism and function at the basic and clinical level. J Endocr Soc. 4:bvz038. doi.org/10.1210/jendso/bvz038.

Blanco-Melo D, Nilsson-Payant BE, Liu W-C, Uhl S, Hoagland D et al. (2020) Imbalanced host response to SARSCoV-2 drives development of COVID-19. Cell 181: 10361045.e9.

Bradshaw PC, Seeds WA, Miller AC, Mahajan VR, Curtis WM (2020) COVID-19: Proposing a ketone-based metabolic therapy as a treatment to blunt the cytokine storm. Oxid Med Cell Longev 6401341. https://doi.org/10.1155/2020/6401341.

Brook CE, Boots M, Chandran K, Dobson AP, Drosten Cet al. (2020) Accelerated viral dynamics in bat cell lines, with implications for zoonotic emergence. eLife 9: e48401. DOI: https://doi.org/10.7554/eLife.48401.

Bouchama A, Aziz MA, Mahri SA, Gabere MN, Dlamy MA et al. (2017) A model of exposure to extreme environmental heat uncovers the human transcriptome to heat stress. Sci Rep 7: 9429. DOI:10.1038/s41598-017-09819-5.

Centre for Disease Control and Prevention (CDC). COVID19 and animals. CDC report (2021). https://www.cdc.gov/ coronavirus/2019-ncov/daily-life-coping/animals.html. Accessed 2 May 2021

Chang C-H, Zhou X-W, Wang Y-C, Lee T-H (2020) Differential effects of hypothermal stress on lactate metabolism in fresh water- and seawater-acclimated milkfish, Chanos chanos. Comp Biochem Physiol A 248: 110744.

Cavaleros M, Buffenstein R, Ross FP, Pettifor JM (2003) Vitamin D metabolism in a frugivorous nocturnal mammal, the Egyptian fruit bat (Rousettus aegyptiacus). Gen Comp Endocrinol 133(1): 109-117.

Clarke NE, Belyaev ND, Lambert DW, Turner AJ (2014) Epigenetic regulation of angiotensin-converting enzyme 2 (ACE2) by SIRT1 under conditions of cell energy stress. Clin Sci 126: 507-516.

Codo AC, Davanzo GG, de Brito Monteiro L, de Souza GF, Muraro SP et al. (2020) Elevated glucose lvels favor SARSCoV-2 infection and monocyte response through a HIF-1a/ glycolysis-dependent axis. Cell Metab, https://doi.org/10.1016/ j.cmet.2020.07.007. 
Demarest TG, McCarthy MM (2015) Sex differences in mitochondrial (dys)function: Implications for neuroprotection. J Bioenerg Biomembr 47(1-2): 173-188. doi: 10.1007/s10863014-9583-7.

Dong Y, Brewer GJ (2019) Global metabolic shifts in age and Alzheimer's disease mouse brains pivot at NAD+/NADH redox sites. J Alzheimers Dis 71(1): 119-140. doi: 10.3233/ JAD-190408.

Ehrlich A, Uhl S, Ioannidis K, Hofree M, Oever BR et al. (2020) The SARS-CoV-2 transcriptional metabolic signature in lung epithelium. SSRN Electronic J, doi: 10.2139/ ssrn.3650499.

Fink R, Borsting CF (2002) Quantitative glucose metabolism in lactating mink (Mustela vison) - effects of dietary levels of protein, fat and carbohydrates. Acta Agric Scand Sect A Anim Sci 52: 34-42.

Fussell J, Son J, Wagner BA, Sarsour EH, Buettner GR et al. (2016) 166 - A lipogenic switch shifts metabolism from glycolysis to mitochondrial respiration during aging. Free Radic Biol Med 100: S83.

Gaignard P, Savouroux S, Liere P, Pianos A, Thérond P et al. (2015) Effect of sex differences on brain mitochondrial function and its suppression by ovariectomy and in aged mice. Endocrinol 156(8): 2893-2904.

Garcia-Alvarez M, Marik P, Bellomo R (2014) Stress hyperlactataemia: present understanding and controversy. Lancet Diabetes Endocrinol 2: 339-347.

Gaskin RS, Estwick D, Peddi R (2001) G6PD deficiency: its role in the high prevalence of hypertension and diabetes mellitus. Ethn Dis 11: 749-754.

Ghaffari S, Roshanravan N, Tutunchi H, Ostadrahimi A, Pouraghaei M et al. (2020) Oleoylethanolamide, a bioactive lipid amide, as a promising treatment strategy for coronavirus/ COVID-19. Arch Med Res 51:464-467.

Gheblawi M, Wang K, Viveiros A, Nguyen Q, Zhong J-C et al. (2020) Angiotensin-converting enzyme 2: SARS-CoV-2 receptor and regulator of the renin-angiotensin system: Celebrating the 20th anniversary of the discovery of ACE2. Circ Res 126: 1456-74.

Gorbunova V, Seluanov A, Kennedy BK (2020) The world goes bats: Living longer and tolerating viruses. Cell Metab 32: 31-43.

Grasselli G, Zangrillo A, Zanella A, Antonelli M, Cabrini L et al. (2020) Baseline characteristics and outcomes of 1591 patients infected with SARS-CoV-2 admitted to ICUs of the Lombardy Region, Italy. JAMA 323: 1574-1581.

Guo X, Li H, Xu H, Woo S, Dong H et al. (2012) Glycolysis in the control of blood glucose homeostasis. Acta Pharm Sin B 2(4): $358-367$.

Hakim AM, Moss G, Scuderi D (1980) The pentose phosphate pathway in brain during development. Biol Neonate 37: 15-21.

Hardtner C, Morke C, Walther R, Wolke C, Lendeckel U (2013) High glucose activates the alternative ACE2/Ang-(17)/Mas and APN/Ang IV/IRAP RAS Axes in pancreatic â-cells. Int J Mol Med 32: 795-804.

Henry BM, Aggarwal G, Wong J, Benoit S, Vikse J et al. (2020) Lactate dehydrogenase levels predict coronavirus disease 2019 (COVID-19) severity and mortality: A pooled analysis. Am J Emerg Med 38: 1722-1726.

Hosie MJ, Epifano I, Herder V, Orton RJ, Stevenson A et al. (2021) Detection of SARS-CoV-2 in respiratory samples from cats in the UK associated with human-to-cat transmission. Vet Rec e247.

Jain A, Chaurasia R, Sengar NS, Singh M, Mahor S et al. (2020) Analysis of vitamin D level among asymptomatic and critically ill COVID-19 patients and its correlation with inflammatory markers. Sci Rep 10: 20191.

Jamerson BD, Haryadi TH, Bohannon A (2020) Glucose-6phosphate dehydrogenase deficiency: An actionable risk factor for patients with COVID-19? Arch Med Res 51(7): 743-744.

John Hopkins University and Medicine, 2021. CORONAVIRUS RESOURCE CENTER. https:// coronavirus.jhu.edu/. Accessed 17 June 2021.

Johnson AA, Stolzing A (2019) The role of lipid metabolism in aging, lifespan regulation, and age-related disease. Aging Cell 18: e13048.

Lanham-New SA, Webb AR, Cashman KD, Buttriss JL, Fallowfield JL et al. (2020) Vitamin D and SARS-CoV-2 virus/ COVID-19 disease. BMJ Nutr Prevent Hlth 3(1): 106-110.

Lazzer S, Bedogni G, Lafortuna CL, Marazzi N, Busti C et al. (2012) Relationship between basal metabolic rate, gender, age, and body composition in 8,780 white obese subjects. Obesity 18 (1): 71-78.

Li M-Y, Li L, Zhang Y, Wang X-S (2020) Expression of the ARS-CoV-2 cell receptor gene ACE2 in a wide variety of human tissues. Infect Dis Poverty 9: 45. 
Liberti MV, Locasale JW (2016) The Warburg effect: How does it benefit cancer cells. Trends Biochem Sci 41(3): 211218.

Liu G, Summer R (2019) Cellular metabolism in lung health and disease. Ann Rev Physiol 81: 403-428.

Marais GAB, Gaillard JM, Vieira C, Plotton I, Sanlaville D et al. (2018) Sex gap in aging and longevity: can sex chromosomes play a role? Biol Sex Differ 9: 33.

Mayer KA, Stockl J, Zlabinger GJ, Gualdoni GA (2019) Hijacking the supplies: Metabolism as a novel facet of virushost interaction. Front Immunol 10: 1533.

Mehrabani S, Bagherniya M, Askari G, Read MI, Sahebkar A (2020) The effect of fasting or calorie restriction on mitophagy induction: a literature review. J Cachexia Sarcopenia Muscle 11(6): 1447-1458.

Melendez-Morales D, de Paz-Lugo P, Melendez-Hevia E (2009) Glycolytic activity in flight muscles of birds according to their physiological function. An experimental model in vitro to study aerobic and anaerobic glycolysis activity separately. Mol Cell Biochem 328: 127-135.

Meng F, Zhu L, Huang W, Irwin DM, Zhang S (2016) Bats: Body mass index, forearm mass index, blood glucose levels and SLC2A2 genes for diabetes. Sci Rep 6: 29960. DOI: $10.1038 /$ srep29960.

Miller B, Silverstein A, Flores M, Cao K, Kumagai H et al. (2021) Host mitochondrial transcriptome response to SARSCoV-2 in multiple cell models and clinical samples. Sci Rep 11: 3 .

Nasr LB, Monet JD, Lucas P, Bader CA (1989) Vitamin D3 and glucose-6-phosphate dehydrogenase in rat duodenal epithelial cells. Am J Physiol 257(5 Pt 1): G760-5.

Niedermuller H (1986) Effects of ageing on the recycling via pentose cycle and on the kinetics of glycogen and protein metabolism in various organs of the rat. Arch Gerontol Geriatr 5(4): 305-316.

Ofusori D, Adelakun A, Adesanya O, Ojo G, Oluyemi K et al. (2007) Comparative evaluation of the activities of LDH and $\mathrm{SDH}$ in the small intestine of pangolin Anis tricuspis and bat Eidolon helvum. Internet J Vet Med 4, https:// www.researchgate.net/publication/344345372.

Pennisi E (2020) How bats have outsmarted virusus including coronaviruses - for 65 million years. Science, doi:10.1126/science. abd9595.
Pinheiro EC, Taddei VA, Migliorini RH, Kettelhut IS (2006) Effect of fasting on carbohydrate metabolism in frugivorous bats (Artibeus lituratus and Artibeus jamaicensis). Comp Biochem Physiol B 143: 279-284.

Pinto BGG, Oliveira AER, Singh Y, Jimenez L, Gonçalves ANA et al (2020) ACE2 expression is increased in the lungs of patients with comorbidities associated with severe COVID-19. J Infect Dis 222: 556-63.

Polak-Iwaniuk A, Harasim-Symbor E, Golaszewska K, Chabowski A (2019) How hypertension affects heart metabolism. Front Physiol 10: 435.

Protzek AOP, Rafacho A, Viscelli BA, Bosqueiro JR, Cappelli AP et al (2010) Insulin and glucose sensitivity, insulin secretion and â-cell distribution in endocrine pancreas of the fruit bat Artibeus lituratus. Comp Biochem Physiol A 157: 142148 .

Rand JS, Kinnaird E, Baglioni A, Blackshaw J, Priest J (2002) Acute stress hyperglycemia in cats is associated with struggling and increased concentrations of lactate and norepinephrine. J Vet Intern Med 16(2): 123-32.

Randeria SN, Thomson GJA., Nell TA, Roberts T, Etheresia Pretorius E (2019) Inflammatory cytokines in type 2 diabetes mellitus as facilitators of hypercoagulation and abnormal clot formation. Cardiovasc Diabetol 18:72.

Rastogi A, Bhansali A, Khare N, Suri V, Yaddanapudi N et al. (2020) Short term, high-dose vitamin D supplementation for COVID-19 disease: a randomised, placebo-controlled, study (SHADE study). Postgraduate Med J 0:1-4.

Ravera S, Podesta M, Sabatini F, Dagnino M, Cilloni D et al. (2019) Discrete changes in glucose metabolism define aging. Sci Rep 9: 10347.

Redavid LA, Sharp CR, Mitchell MA, Beckel NF (2016) Hyperlactatemia and serial lactate measurements in sick cats. J Vet Emerg Crit Care (San Antonio) 26(4): 495-501.

Santos GC, Zeidler JD, Pérez-Valencia JA, Sant'Anna-Silva ACB, Da Poian AT et al. (2017) Metabolomic analysis reveals vitamin D-induced decrease in polyol pathway and subtle modulation of glycolysis in HEK293T cells. Sci Rep 7: 9510.

Schermerhorn T (2013) Normal glucose metabolism in carnivores overlaps with diabetes pathology in non-carnivores. Front Endocrinol 4: 188.

Silaidos C, Pilatus U, Grewal R, Matura S, Lienerth B et al. (2018) Sex-associated differences in mitochondrial function in 
human peripheral blood mononuclear cells (PBMCs) and brain. Biol Sex Differ 9: 34.

Singh KK, Chaubey G, Chen JY, Suravajhala P (2020) Decoding SARS-CoV-2 hijacking of host mitochondria in COVID-19 pathogenesis. Am J Physiol Cell Physiol 319: C258C267.

Smith SM, Boppana A, Traupman JA, Unson E, Maddock DA et al. (2020) Impaired glucose metabolism in patients with diabetes, prediabetes and obesity is associated with severe COVID-19. J Med Virol 93(1): 409-415.

Sullivan KM, Somero GN (1980) Enzyme activities of fish skeletal muscle and brain as influenced by depth of occuerence and habits of feeding and locomotion. Mar Biol 60: 91-99.

Sun J, Liu Q, Zhao L, Cui C, Wu H et al. (2019) Potential regulation by miRNAs on glucose metabolism in liver of common carp (Cyprinus carpio) at different temperatures. Comp Biochem Physiol D 32: 100628.

Timper K, Denson JL, Steculorum SM, Heilinger C, Engstrom-Rudd L et al. (2017) IL-6 improves energy and glucose homeostasis in obesity via enhanced central IL-6 transsignaling. Cell Rep 19:267-280.

Thomas SP, Suthers RA (1972) The physiology and energetics of bat flight. J Exp Biol 57: 317-335.

Torchon E, Ray R, Hulver MW, McMillan RP, Voy BH (2017) Fasting rapidly increases fatty acid oxidation in white adipose tissue of young broiler chickens. Adipocyte 6(1):3339.

Uranga RM, Keller JN (2019) The complex interactions between obesity, metabolism and the brain. Front Neurosci 13: 513.

van Beek JHGM, Kirkwood TBL, Bassingthwaighte JB (2016) Understanding the physiology of the ageing individual:
Computational modelling of changes in metabolism and endurance. Interface Focus 6: 20150079.

Vick DJ (2020) Glucose-6-phosphate dehydrogenase deficiency and COVID-19 infection. Mayo Clin Proc 95: 17971809.

Washizu T, Kuramoto E, Abe M, Sako T, Arai T (1998) A comparison of the activities of certain enzymes related to energy metabolism in leukocytes in dogs and cats. Vet Res Commun 22: 187-192.

Wong SCC, Chan JKC, Lee KC, Lo ESF, Tsang DNC (2005) Development of a quantitative assay for SARS coronavirus and correlation of GAPDH mRNA with SARS coronavirus in clinical specimens. J Clin Pathol 58: 276-280.

Wu Y-H, Tseng C-P, Cheng M-L, Ho H-Y, Shih S-R et al. (2008) Glucose-6-phosphate dehydrogenase deficiency enhances human coronavirus 229E infection. J Infect Dis 197: 812-816.

Wu D, Shu T, Yang X, Song J-X, Zhang M et al. (2020) Plasma metabolomic and lipidomic alterations associated with COVID-19. Nat Sci Rev 7: 1157-1168.

Xie X, Chen J, Wang X, Zhang F, Liu Y (2006) Age- and gender-related difference of ACE2 expression in rat lung. Life Sci 78: 2166-71.

Yang Y, Xiong S, Cai B, Luo H, Dong E et al. (2017) Mitochondrial $\mathrm{C} 11$ orf33 is a potent antiviral protein independent of interferon production. Sci Rep 7: 44303.

Zeng Z, Yu X, Ziao S, Yao H, Zhu J (2017) Effects of 1,25dihydroxyvitamin D3 on pathological changes in rats with diabetic cardiomyopathy. Lipids Health Dis 16: 109.

Zhou P, Tachedjian M, Wynne JW, Boyd V, Cui J et al. (2016) Contraction of the type I IFN locus and unusual constitutive expression of IFN-á in bats. Proc Natl Acad Sci USA 113: 26962701.

*Cite this article as: Manna SK (2021) Novel coronavirus vulnerability: Metabolic derangements and altered dehydrogenase enzyme activities are central to the virus pathogenesis in human, bat and other animals. Explor Anim Med Res 11(1): 1-9. DOI : 10.52635/EAMR/11.1.1-9 